\title{
Interstitial Cells of Cajal: Potential Targets for Functional Dyspepsia Treatment Using Medicinal Natural Products
}

\author{
Jin-Yong Joung $\mathbb{D},{ }^{1}$ Seo-Hyung Choi $\mathbb{D}^{2},{ }^{2}$ and Chang-Gue Son $\mathbb{D}^{1}$ \\ ${ }^{1}$ Liver and Immunology Research Center, Oriental Medical College of Daejeon University, 75, Daedeok-daero 176 beon-gil, \\ Seo-gu, Daejeon 35235, Republic of Korea \\ ${ }^{2}$ Department of Internal Medicine, Weedahm Oriental Hospital, 430, Yeoksam-ro, Gangnam-gu, Seoul 06200, Republic of Korea \\ Correspondence should be addressed to Chang-Gue Son; ckson@dju.ac.kr
}

Received 24 March 2021; Accepted 25 June 2021; Published 30 June 2021

Academic Editor: Fadia S. Youssef

Copyright (c) 2021 Jin-Yong Joung et al. This is an open access article distributed under the Creative Commons Attribution License, which permits unrestricted use, distribution, and reproduction in any medium, provided the original work is properly cited.

\begin{abstract}
Introduction. The pathophysiology of functional dyspepsia (FD) remains uncertain, but the interstitial cells of Cajal (ICCs), pacemakers that regulate gastrointestinal motility, are garnering attention as key modulators and therapeutic targets in FD. This review comprehensively discusses the involvement of ICCs in the pharmacologic actions of FD and as therapeutic targets for herbal products for FD. Methods. A search of the literature was performed using PubMed by pairing "interstitial cells of Cajal" with "medicinal plant, herbal medicine, phytotherapy, flavonoids, or traditional Chinese medicine (TCM)." Results. From the 55 articles screened in the initial survey, 34 articles met our study criteria. The search results showed that herbal products can directly depolarize ICCs to generate pacemaker potentials and increase the expression of c-kit and stem cell factors, helping to repair ICCs. Under certain pathological conditions, medicinal plants also protect ICCs from oxidative stress and/or inflammation-induced impairment. Two representative herbal decoctions (Banhasasim-tang, 半夏泻心汤, and Yukgunja-tang, 六君子汤) have been shown to modulate ICC functions by both clinical and preclinical data. Conclusion. This review strongly indicates the potential of herbal products to target ICCs and suggests that further ICC-based studies would be promising for the development of FD treatment agents.
\end{abstract}

\section{Introduction}

Functional dyspepsia (FD) is one of the most common gastrointestinal (GI) disorders, affecting $10-30 \%$ of the population worldwide [1]. It is characterized as a recurrent or persistent disorder of sensation and movement in the upper digestive tract without any explainable organic causes [2]. Although FD is not generally life threatening, it is known to impair physical, mental, and social aspects of quality of life. The socioeconomic burden of FD was estimated at US\$ 18.4 billion in the USA in 2009 [3].

The underlying pathophysiology of FD is not yet fully understood, but Helicobacter pylori infection, visceral hypersensitivity, acid disorders, psychosocial factors, and/or abnormal gut motility are considered to be the main contributors to FD [4]. Given these pathological factors,
H. pylori eradication, proton pump inhibitors (PPIs), histamine-type-2 receptor antagonists, tricyclic antidepressants (TCAs), and prokinetic drugs are widely used for patients with FD [5]. However, these agents have shown relatively low response rates and frequent relapse at rates of up to $73 \%$, which leads to clinical limitations [6]. Therefore, researchers are looking for new targets for the treatment of FD [7].

As key modulators of the pathophysiology of FD, interstitial cells of Cajal (ICCs) are receiving attention. ICCs are a type of interstitial cell found in the GI tract and are known to play a major role in GI motility [8]. ICCs mediate input from the GI motor nervous system to smooth muscle and act as pacemakers in GI motility by generating spontaneous electrical slow waves to stimulate rhythmic peristalsis. Recently, increasing evidence has suggested that loss or dysfunction of ICCs is a cause of GI motility disorders, 
especially FD [9-11]. Thus, ICCs are currently considered targets for pharmacological intervention for patients with FD.

Herbal products have been used as treatment options for patients with GI disorders. One study reported that $34.7 \%$ of surveyed patients with functional GI disorders used herbal medicines [12]. Many researchers are also investigating herbal medicines that can modulate multiple targets via their multiple active components. Most herb-derived benefits for GI disorders are thought to be linked to GI motility [13]. Herbal medicines are also proposed to affect the functions of ICCs, which have complex interactions with surrounding cells and express various receptors for neurotransmitters and circulating hormones $[11,14]$.

This review aims to analyze the current status and evidence for medicinal natural products related to FD treatment that target ICCs and to increase knowledge regarding ICCs in the context of FD.

\section{Strategy for Literature Survey and Overall Features of Results}

We conducted a literature search using three using PubMed (http://www.ncbi.nlm.nih.gov/pubmed) by pairing "interstitial cells of Cajal" with "herbal medicine, phytotherapy, flavonoids, or traditional Chinese medicine." The search was conducted on papers published until November 2020.

From the 55 articles screened by the initial survey, a total of 34 related articles were selected, of which 13 described in vivo studies, 18 described in vitro studies, and 3 described both in vivo and in vitro studies. Among those studies, 22 used herbal prescriptions, 4 used herbs, and 8 used flavonoids. Banhasasim-tang (半夏泻心汤), a traditional Chinese medicine, was the most frequently studied compound and was used in 3 studies.

\section{Overview of ICC Physiology}

ICCs, discovered by Santiago Ramón y Cajal in 1911, are known as end structures of the intrinsic nervous system in the GI tract that mediate nerves and smooth muscle cells [15]. ICCs connect with other ICCs and smooth muscle cells through gap junctions, forming a syncytium in GI tissue [16]. ICCs were first shown to play a key role in GI motility by generating slow waves that induce rhythmic contraction in smooth muscles by Faussone Pellegrini in 1977 [17]. The most widely known mechanism for slow wave generation involves intracellular changes in $\mathrm{Ca}^{2+}$ concentrations inside ICCs [11]. In this mechanism, the release of $\mathrm{Ca}^{2+}$ into the cytoplasm from the endoplasmic reticulum (ER) is first initiated by the ignition of ryanodine (RYR) receptors and/ or inositol triphosphate 3 (IP3) receptors. In response to this, overabsorption of $\mathrm{Ca}^{2+}$ into the mitochondria occurs, resulting in a localized decrease in the $\mathrm{Ca}^{2+}$ concentration. Next, $\mathrm{Ca}^{2+}$-inhibited nonselective cation ion channels are activated, leading to rapid cellular influx of $\mathrm{Ca}^{2+}$ from the extracellular space, which is called spontaneous transient depolarization (STD). The STD signal is delivered to the GI smooth muscles through gap junctions and causes smooth muscle contractions (Figure 1). Recently, researchers have found other membrane channels involved in regulating the intracellular $\mathrm{Ca}^{2+}$ concentration, including anoctamin 1 (ANO1, a calcium-activated chloride channel) and transient receptor potential melastatin 7 (TRPM 7) [10, 18].

The slow-wave potentials in ICCs respond to both neural and nonneural inputs under physiological and/or pathological conditions [19]. ICCs are innervated by myenteric nerves that act as key neural stimulators for GI motility [11]. ICCs act via various receptors for neurotransmitters and circulating hormones. To date, muscarinic acetylcholine receptors (mainly $\mathrm{M}_{3}$ receptors), serotonin (5-HT) receptors (mainly $5-\mathrm{HT}_{3}$ and $5-\mathrm{HT}_{3}$ receptors), and $\mathrm{G}$ protein-coupled receptors have been the relatively well studied $[15,20,21]$ (Figure 2).

$M_{3}$ and $M_{2}$ receptors are important in neuronal signaling from enteric motor neurons [15]. In this process, phospholipase C (PLC) and IP3 act as key molecules for the transformation of signals into ER-derived $\mathrm{Ca}^{2+}$ oscillations, leading to depolarization [22]. Serotonergic stimulation, one of the important contributors to the brain-gut connection, also modulates intracellular $\mathrm{Ca}^{2+}$ in ICCs and generates slow-wave potentials through $5-\mathrm{HT}_{3}$ and/or $5-\mathrm{HT}_{4}$ receptors. The underlying mechanisms may involve induction of $\mathrm{Ca}^{2+}$ influx from the extracellular space through 5-HT receptors and simultaneous activation of voltage-gated $\mathrm{Ca}^{2+}$ permeable channels [23]. Ghrelin, known as a hunger hormone, is secreted by enteroendocrine cells and increases food intake. It binds to $G$ protein-coupled receptors and regulates intracellular and extracellular $\mathrm{Ca}^{2+}$ concentrations in ICCs, leading to the generation of pacemaker potential via $\mathrm{IP}_{3^{-}}$, Rho kinase-, and protein kinase C (PKC)-dependent signaling pathways [24]. In contrast, certain neurotransmitters or hormones can inhibit pacemaker potential in ICCs via nitric oxide/cyclic guanosine monophosphate $(\mathrm{NO} /$ cGMP) signaling pathways or noradrenaline-induced stimulation of $\beta_{1}$-adrenoceptors [25, 26].

On the other hand, ICCs also have c-kit, a tyrosine kinase receptor that plays an essential role in the development of ICCs by binding with its ligand, stem cell factor (SCF) [27]. In general, immunohistochemistry of anti-c-kit can be used to examine the distribution and density of ICCs and can also be used to identify the structure of ICCs [28].

\section{Pathophysiologic Involvement of ICC in FD}

Based on the functions of ICCs in generating slow-wave potentials for GI movement, ICC dysfunction can cause many GI motility disorders [29]. FD is known as one of the representative diseases associated with dysfunction of ICCs [30]. Abnormal gastric slow waves have been observed in patients with FD via multichannel electrogastrography [31]. Pathological findings have shown that delayed gastric dysmotility is associated with degeneration of ICCs in both humans [32] and animals [33]. In an FD rat model induced by chronic stress via tail clamping, the levels of autophagic biomarkers in ICCs were increased, whereas the levels of differentiation biomarkers in ICCs, such as c-kit and SCF, were decreased [30]. Although the relationship between ICC 


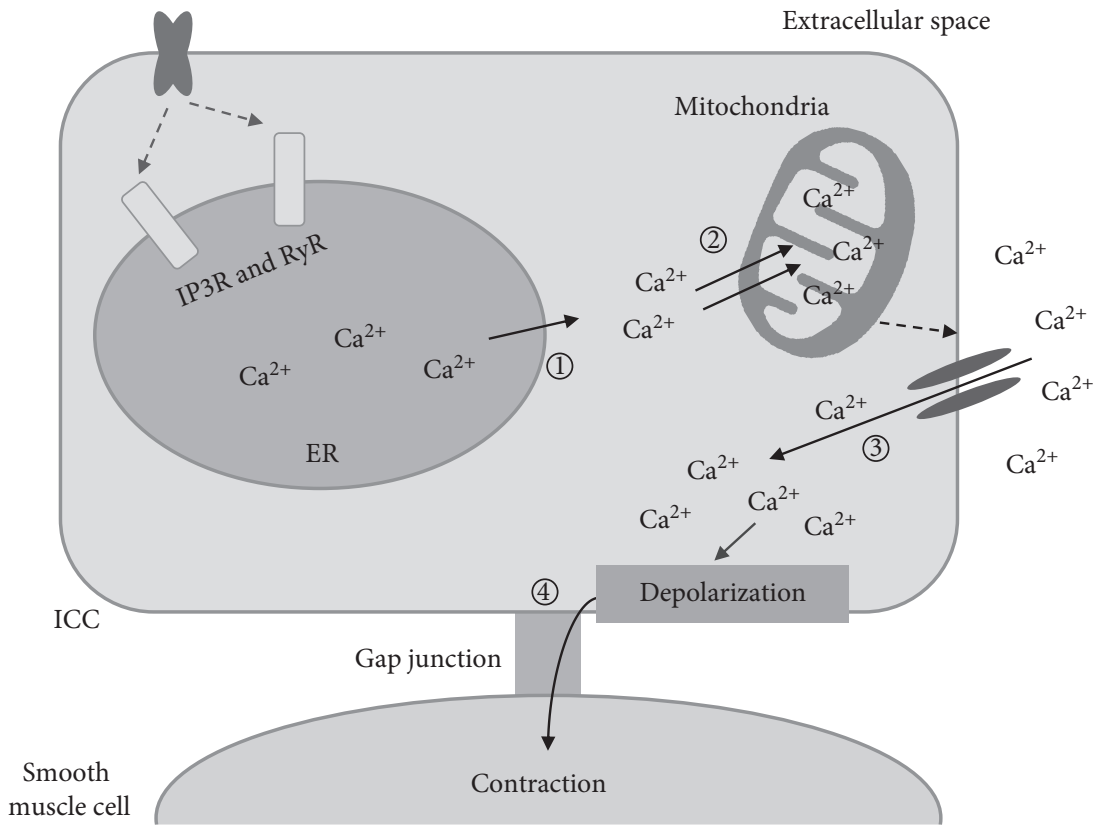

Figure 1: Mechanism for the generation of slow-wave potentials in ICCs. (1) The release of $\mathrm{Ca}^{2+}$ into the cytoplasm from the endoplasmic reticulum (ER) is initiated by the activation of ryanodine (RYR) receptors and/or inositol triphosphate 3 (IP3) receptors. (2) Overabsorption of $\mathrm{Ca}^{2+}$ into the mitochondria occurs, resulting in a localized drop in $\mathrm{Ca}^{2+}$ concentration. (3) $\mathrm{Ca}^{2+}$-inhibited nonselective cation ion channels are activated, leading to rapid cellular influx of $\mathrm{Ca}^{2+}$ from the extracellular space, which produces spontaneous transient depolarizations (STDs). (4) STDs are delivered to the GI smooth muscles through gap junctions and cause smooth muscle contractions.

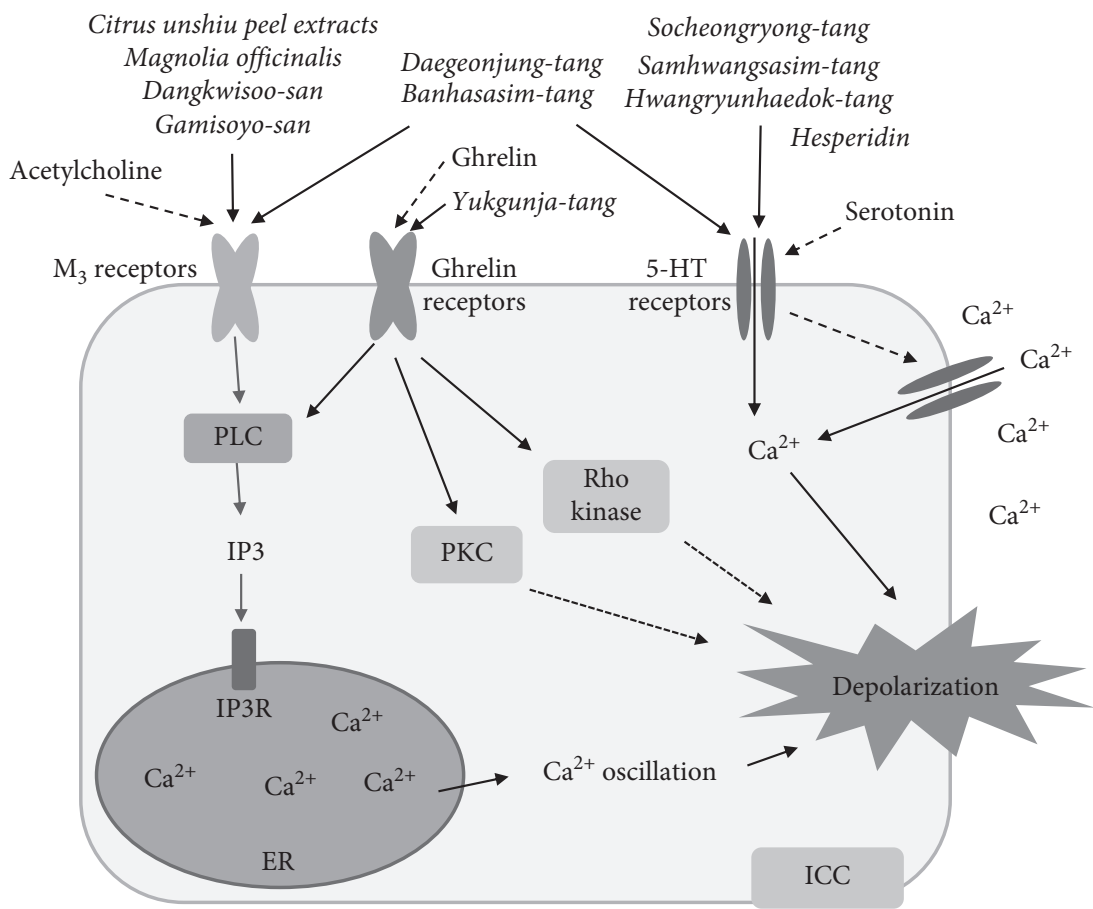

Figure 2: Pathways of the generation of slow-wave potentials by herbal medicines.

dysfunction and FD has not been accurately elucidated, excessive autophagy and abnormal differentiation of ICCs seem to contribute to the pathogenesis of FD [11].

In addition, patients with Crohn's disease [34], ulcerative colitis [35], and chronic bowel obstruction tend to have low
ICC counts [36]. Animal studies have reported impaired function of ICCs under proinflammatory conditions $[37,38]$. Consistent with these findings, functional recovery or regeneration of ICCs can help ameliorate digestive disorders. In several artificial GI dysmotility rodent models, 
positive correlations between improvements in ICC structure and GI motility have been found $[33,39,40]$. These data indicate the importance of ICC impairment or dysfunction in pathophysiology and as a therapeutic target, especially in GI motility-associated disorders, including FD.

\section{Effects of Herbal Products on ICC Receptors and ICC Integrity}

Herbal products can directly depolarize ICCs and generate slow-wave potentials. These results have been obtained mainly from cell-based experiments with ICCs using the patch-clamp technique. Many single-herb extracts [41, 42], TCM drugs [14, 26, 43-51], and herb-derived flavonoids [52] depolarize the pacemaker potential of ICCs in a dose-dependent manner. Depending on the herbal products, different receptors and their signaling pathways are involved in this depolarization process. Three receptors, acetylcholine muscarinic $\mathrm{M} 2 / \mathrm{M} 3$ receptors, $5-\mathrm{HT}_{3} / 5-\mathrm{HT}_{4}$ receptors, and ghrelin receptors, have been the main focus of the related studies (Table 1 and Figure 2). Compounds containing these herbal products are believed to act as ligands for the receptors above in cell culture conditions.

Herbal medicines are also able to activate c-kit and SCF, which are involved in central pathways in the growth of ICCs. Banhasasim-tang, a typical herbal decoction for patients with FD, has been found to significantly promote ICC and SCF signaling in stomach tissues in both a loperamideinduced FD mouse model and a diabetic dyspepsia rat model $[53,54]$. These herbal effects on ICCs and SCF have also been reported in other animal models, including in a trinitrobenzene sulfonic acid-induced rat colitis model treated with Shenqing recipe [40], a cirrhotic ascites rat model treated with Xiaozhang Tie [55], and a loperamide-induced constipation rat model treated with Herba Cistanche [56]. A single flavonoid from citrus fruits and tomatoes, naringenin, has also activated c-kit and SCF, favoring constipation, in a loperamide-induced constipation model [57]. As expected, some herbal drugs (Tong Bian decoction, Jianpi Qingchang decoction, Chaihu Shugan powder, Aurantii Fructus Immaturus, and Atractylodis Macrocephalae Rhizoma) inhibit autophagy of ICCs or alleviate their dysfunction in GI inflammation animal models [58-61]. A summary of the effects of herbal products on ICC integrity is presented in Table 2.

\section{Effects of Herbal Products against Oxidative Stress-Induced ICC Impairment}

Oxidative stress, one of the most common pathological contributors, is also well known to cause both dysfunction and loss of ICCs in the GI tract [62]. Overproduction of NO after surgery has been found to disrupt both GI motility and the slow-wave potentials of ICCs in a mouse model [63]. Cotreatment with interferon-gamma and lipopolysaccharide (IFN- $\gamma+$ LPS) impairs the function and structure of ICCs in tissue culture conditions using the smooth muscle layer of the murine jejunum, while antioxidants and inhibitors of NO synthesis protect against these alterations [64]. In fact, a relatively low concentration of $\mathrm{NO}$, as a neurotransmitter, is essential for ICCs to function properly [65], whereas a higher concentration or long-term exposure to $\mathrm{NO}$ can induce ICC damage [66]. The high density of mitochondria in ICCs can explain the increased susceptibility to oxidative stress conditions [67, 68]. As mentioned above, mitochondria are essential for $\mathrm{Ca}^{2+}$ handling within ICCs to generate slow waves [69].

On the other hand, many herb-related studies have revealed antioxidant and anti-inflammatory properties that result in protection against or treatment of GI disorders, including FD [70, 71]. Oxidative stress and inflammation coexist in most pathologic conditions [72]. Curcumin, a polyphenol from Curcuma longa (turmeric), increases SCF/ c-kit protein levels in stomach tissues and ameliorates diabetic gastroparesis in rats by blocking the development of oxidative stress [73]. Quercetin, a plant flavonol, restores the density of ICCs in the jejuna of diabetic rats through its antioxidant action [74]. Disruption of GI motility is one of the complications of diabetic mellitus, and its link to ICCs has also been clearly reported [75]. In addition, FD and diabetic gastroparesis have similar symptoms and share a very similar pathogenesis [76].

ICC dysfunction or a reduction in ICC numbers does not mean permanent loss. ICCs have a high degree of plasticity and can be transformed into a smooth muscle-like phenotype rather than be completely destroyed [77]. Many herb-related studies have shown that ICCs can recover their original phenotype and function when oxidative stress or inflammation is alleviated in a tail clasping stress rat model $[33,78]$. These facts suggest that the antioxidant activities of herbal products are key characteristics for reversal of ICC dysfunction under oxidative stress conditions, such as FD.

\section{Two Representative TCMs Modulating ICC: Banhasasim-Tang (半夏泻心汤) and Yukgunja-Tang (六君子汤)}

In clinical practice, the most commonly used TCMs for FD are Banhasasim-tang (半夏泻心汤, known as Ban Xia Xie Xin decoction in China and Hange-shashin-to in Japan) and Yukgunja-tang (六君子汤, known as Liu Jun Zi decoction in China and Rikkunshito in Japan). Due to their widespread use, these two decoctions have been widely studied in both clinical and preclinical studies [79, 80]. Clinical practice guidelines for FD published in Japan recommend the use of Yukgunja-tang as a second-line treatment with evidence level A [81].

Banhasasim-tang has been widely prescribed for patients with FD. Several clinical studies have shown the beneficial effects of BST against gastric dyspepsia $[82,83]$, and a metaanalysis study reported that Banhasasim-tang is as effective as conventional treatments [84]. Regarding its underlying mechanisms, several preclinical studies have suggested an association with the recovery of ICCs. Banhasasim-tang depolarizes the slow-wave potentials in ICCs by regulating internal and external $\mathrm{Ca}_{2}{ }^{+}$via the $\mathrm{M}_{3}$ and $5-\mathrm{HT}_{3}$ receptors in a dose-dependent manner [44]. We also found that 
TABLE 1: Medicinal plants acting on the depolarization of ICCs.

Medicinal plants or decoctions (reference)

Depolarizers of the pacemaker potential of ICCs

Gamisoyo-san [14], Citrus unshiu peel extracts [41], Dangkwisoo-san [43]

Magnolia officinalis [42]

Banhasasim-tang [44]

Daegeonjung-tang [45]

Hesperidin, a citrus flavonoid [52]

Hwangryunhaedok-tang [46], Samhwangsasim-tang [47], Socheongryong-tang [48]

Yukgunja-tang [49]

Leejung-tang [90], Sengmek-san [50]

Pyungwi-san [51]

Inhibitors of the pacemaker potential of ICCs

Galgeun-tang [26]

Quercetin [91]

M receptors: muscarinic acetylcholine receptors; 5-HT receptors: 5-hydroxytryptamine receptors; PLC: phospholipase C; PKC: protein kinase C.
Molecular mechanism

Via $\mathrm{M}_{3}$ receptor

Via $\mathrm{M}_{2}$ and $\mathrm{M}_{3}$ receptors

Via $\mathrm{M}_{3}$ and $5-\mathrm{HT}_{3}$ receptors

Via $\mathrm{M}_{3}$ and $5-\mathrm{HT}_{4}$ receptors

Via $5-\mathrm{HT}_{4}$ receptor

Via $5-\mathrm{HT}_{3}$ and $5-\mathrm{HT}_{4}$ receptor

Via ghrelin receptor

Via PLC pathway

Via PKC pathway
Via $\alpha 2$ - and $\beta 1$-adrenoceptors Via opioid receptor signaling pathways

TABLE 2: Medicinal plants acting on the behaviors of ICCs.

\begin{tabular}{lc}
\hline Herbal product & Effect (experimental model) \\
\hline Jianpi Qingchang decoction [60] & Regulation of intestinal motility of DSS-induced colitis \\
(in vivo)
\end{tabular}

Tong bian decoction [58]

Shuwei decoction [33]

Da-Cheng-Qi decoction combined with Lactobacillus acidophilus [39]

Shenqing recipe [40]

Chaihu Shugan powder [61]

Xiaozhang Tie [55]

Herba Cistanche [56]

Total phenols of Magnolia officinalis

Rehd. et Wils. [92]

Aurantii Fructus Immaturus and

Atractylodis Macrocephalae

Rhizoma [59]

Eugenol and cinnamaldehyde

(transdermal administration) [93]

Aconitine, emodin [94]

Hesperidin, a citrus flavonoid [52]

Naringenin [57]

Quercetin [74]

Nobiletin [95]

Eugenol* $^{*}$ [96]
Enhancement of colon transport function (in vivo) Improvement of gastric motility in a tail claspinginduced functional dyspepsia rat model (in vivo)

Improvement of GI motility in mice with traumatic brain injury (in vivo)

Repair of the ultrastructure of colonic ICCs in a TNBSinduced colitis rat model (in vivo)

Inhibition of excessive autophagy of ICCs (in vitro)

Reduction in the degree of ascites and improvement of intestinal motility in cirrhotic rats (in vivo)

Improvement of loperamide-induced slow transit constipation (in vivo)

Improvement of gastric motility in an atropineinduced GI dysmotility rat model (in vivo)

Protection of glutamic acid-stimulated ICCs (in vitro)

Increase in ICC numbers in a trinitrobenzene sulfonate-induced ulcerative colitis rat model (in vivo)

Toxicity toward ICC cells individually but not in combination (aconitine:emodin as $2: 1$ ) (in vitro)

Increase in GI motility, depolarization of the pacemaker potentials of ICCs (in vivo and in vitro) Improvement of loperamide-induced constipation (in vivo)

Increase in ICC numbers in a diabetic rat model (in vivo)

Induction of contraction in weakly contractile states, inhibition of contraction in highly contractile states (in vitro)

Inhibition of intestinal contractions (in vitro)

\footnotetext{
* Drug inhibiting the function of ICCs.
}

Molecular mechanism

IL-10, IFN- $\gamma$, c-kit mRNA $\uparrow \uparrow$

TNF- $\alpha$, IL-1, LC3-II, Beclin-1, NF- $\kappa$ B p65 mRNA $\beta \downarrow \downarrow$

C-kit, nNOS, 5HT4R, ANO1, RYR3 and smMLCK gene $\uparrow \uparrow$

$$
\text { ICCs, SCF } \uparrow \uparrow
$$

ICC, c-kit mRNA $\uparrow \uparrow$

Serum SCF $\uparrow \uparrow$, serum NO $\downarrow \downarrow$, improvement of the structure of ICCs Improvement of ICC network structure damage

C-kit $\uparrow \uparrow$

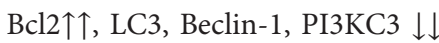

$$
\text { SCF, c-kit } \uparrow \uparrow
$$

C-kit, SCF, PI3K $\uparrow \uparrow$,

$$
\text { SCF, c-kit } \uparrow \uparrow
$$

Reduction in autophagy via inhibition of the PI3K/Akt/mTOR pathway

$$
\text { SCF, c-kit } \uparrow \uparrow
$$

Deactivation of the $\mathrm{Na}^{+} / \mathrm{K}^{+}$-ATPase pump

Via $5-\mathrm{HT}_{4}$ receptor

C-Kit, SCF, aquaporin $3 \uparrow \uparrow$

(possibly due to its antioxidant action)

Via c-kit-dependent pathway

Inhibition of $\mathrm{Ca}^{2+}$-activated $\mathrm{Cl}^{-}$ channel TMEM16A in ICCs 


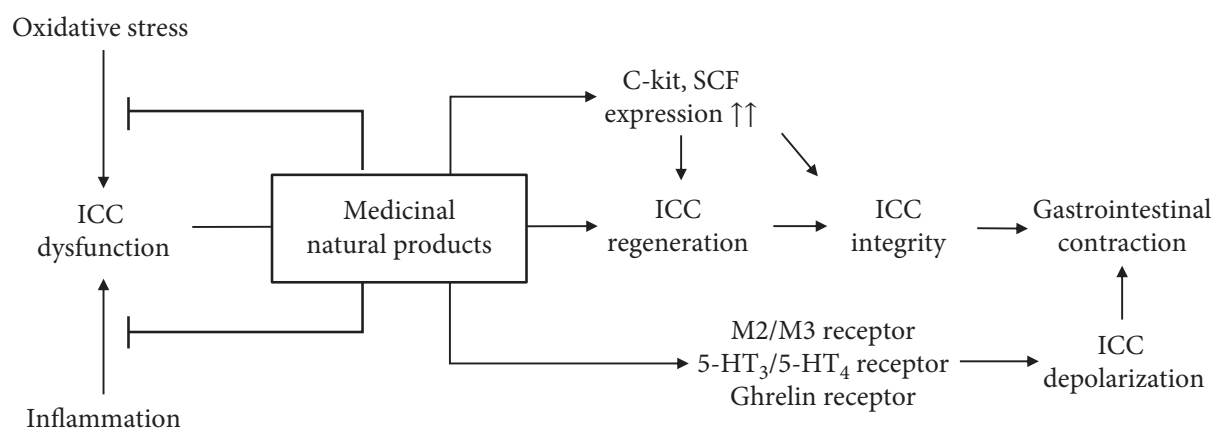

Figure 3: Protective or restorative effects of herbal medicines against ICC dysfunction.

Banhasasim-tang can reverse the inactivation of c-kit and modulate oxidative stress and contraction-related genes such as the $5-\mathrm{HT}_{4}$ receptor, ANO1, RYR receptor 3, and smooth muscle myosin light chain kinase in a loperamideinjected mouse model [53]. This decoction also promotes the numbers of stomach ICCs and the levels of SCF in diabetic rats [54].

Yukgunja-tang has also been frequently investigated for its usefulness in FD treatment using both clinical and preclinical studies. Several RCTs have reported the positive effects of Yukgunja-Tang for FD treatment $[85,86]$. A metaanalysis analyzed 52 RCTs, and Yukgunja-tang was a strong therapeutic for FD management in patients [87]. The therapeutic mechanisms of Yukgunja-tang are known to involve gastric ghrelin secretion, which promotes ICC depolarization in the GI tract [24]. Yukgunja-tang administration increases the plasma levels of ghrelin in both healthy subjects [88] and FD patients [86]. Yukgunja-tang also acted directly on the ghrelin receptor of ICCs and then depolarized the pacemaker potential in an ICC culture experiment [49]. In addition, the strong antioxidative effects of Yukgunjatang in the GI tract have been evidenced in animal studies $[80,89]$, which may contribute to the amelioration of ICC impairment.

\section{Conclusion}

Accumulated evidence has revealed the effects of herbal products on ICCs. These products can increase ICC numbers or restore ICC function. Their therapeutic actions can be categorized as follows: first, they can modulate ICC receptors via ligand-like actions; second, they can protect or restore ICC integrity via activation of c-kit; and third, they can exert antioxidant effects to protect against or reverse ICC dysfunction (Figure 3 ). The available data focusing on the effects of herbal products on ICCs sufficiently support the importance of ICCs as therapeutic targets of herb-derived treatments for FD. Our study has some limitations, such as a lack of human-derived direct evidence and a lack of identification of active compounds, especially those in decoctions such as Banhasasim-tang and Yukgunja-tang. Nevertheless, our present study suggests that promising new drugs can be developed for FD using herbal resources.

\section{Conflicts of Interest}

The authors declare that they have no conflicts of interest.

\section{Acknowledgments}

This study was supported by the Ministry of Education, Science, and Technology (NRF-2018R1A6A1A03025221) and the National Research Foundation of Korea (2019R1A2C2088201).

\section{References}

[1] P. Enck, F. Azpiroz, G. Boeckxstaens et al., "Functional dyspepsia," Nature Reviews Disease Primers, vol. 3, no. 1, pp. 1-20, 2017.

[2] P. Aro, N. J. Talley, L. Agréus et al., "Functional dyspepsia impairs quality of life in the adult population," Alimentary Pharmacology \& Therapeutics, vol. 33, no. 11, pp. 1215-1224, 2011.

[3] B. E. Lacy, K. T. Weiser, A. T. Kennedy, M. D. Crowell, and N. J. Talley, "Functional dyspepsia: the economic impact to patients," Alimentary Pharmacology \& Therapeutics, vol. 38, no. 2, pp. 170-177, 2013.

[4] S. Futagami, M. Shimpuku, Y. Yin et al., "Pathophysiology of functional dyspepsia," Journal of Nippon Medical School, vol. 78, no. 5, pp. 280-285, 2011.

[5] A. C. Ford, S. Mahadeva, M. F. Carbone, B. E. Lacy, and N. J. Talley, "Functional dyspepsia," The Lancet, vol. 396, no. 10263, pp. 1689-1702, 2020.

[6] N. Talley and M. Walker, "Emerging evidence that irritable bowel syndrome \& functional dyspepsia are microbial diseases," Indian Journal of Medical Research, vol. 149, no. 4, pp. 437-440, 2019.

[7] G. Chiarioni, M. Pesce, A. Fantin, and G. Sarnelli, "Complementary and alternative treatment in functional dyspepsia," United European Gastroenterology Journal, vol. 6, no. 1, pp. 5-12, 2018.

[8] D. Al-Sajee and J. D. Huizinga, "Interstitial cells of cajal: pathology, injury and repair," Sultan Qaboos University Medical Journal, vol. 12, no. 4, pp. 411-421, 2012.

[9] N. Zárate, F. Mearin, X.-Y. Wang et al., "Severe idiopathic gastroparesis due to neuronal and interstitial cells of Cajal degeneration: pathological findings and management," Gut, vol. 52, no. 7, pp. 966-970, 2003.

[10] J. Forster, I. Damjanov, Z. Lin, I. Sarosiek, P. Wetzel, and R. Mccallum, "Absence of the interstitial cells of Cajal in patients with gastroparesis and correlation with clinical 
findings," Journal of Gastrointestinal Surgery, vol. 9, no. 1, pp. 102-108, 2005.

[11] O. Al-Shboul, "The importance of interstitial cells of cajal in the gastrointestinal tract," Saudi Journal of Gastroenterology, vol. 19, no. 1, pp. 3-15, 2013.

[12] E. Lahner, S. Bellentani, R. D. Bastiani et al., "A survey of pharmacological and nonpharmacological treatment of functional gastrointestinal disorders," United European Gastroenterology Journal, vol. 1, no. 5, pp. 385-393, 2013.

[13] H. Suzuki, J. M. Inadomi, and T. Hibi, "Japanese herbal medicine in functional gastrointestinal disorders," Neurogastroenterology \& Motility, vol. 21, no. 7, pp. 688-696, 2009.

[14] D. Kim, J. N. Kim, J. H. Nam, J. R. Lee, S. C. Kim, and B. J. Kim, "Modulation of pacemaker potentials in murine small intestinal interstitial cells of Cajal by gamisoyo-san, a traditional Chinese herbal medicine," Digestion, vol. 98, no. 1, pp. 56-68, 2018.

[15] S. M. Ward, E. A. H. Beckett, X. Wang, F. Baker, M. Khoyi, and K. M. Sanders, "Interstitial cells of Cajal mediate cholinergic neurotransmission from enteric motor neurons," The Journal of Neuroscience, vol. 20, no. 4, pp. 1393-1403, 2000.

[16] T. Komuro, "Structure and organization of interstitial cells of Cajal in the gastrointestinal tract," The Journal of Physiology, vol. 576, no. 3, pp. 653-658, 2006.

[17] J. D. Huizinga, J. Chen, H. B. Mikkelsen et al., "Interstitial cells of Cajal, from structure to function," Frontiers in Neuroscience, vol. 7, 2013.

[18] B. J. Kim, J. H. Nam, and S. J. Kim, "Effects of transient receptor potential channel blockers on pacemaker activity in interstitial cells of cajal from mouse small intestine," Molecules and Cells, vol. 32, no. 2, pp. 153-160, 2011.

[19] K. M. Sanders, S. D. Koh, and S. M. Ward, "Interstitial cells of cajal as pacemakers in the gastrointestinal tract," Annual Review of Physiology, vol. 68, no. 1, pp. 307-343, 2006.

[20] G. M. Mawe and J. M. Hoffman, "Serotonin signalling in the gut-functions, dysfunctions and therapeutic targets," Nature Reviews Gastroenterology \& Hepatology, vol. 10, no. 8, pp. 473-486, 2013.

[21] S. Iino and K. Horiguchi, "Interstitial cells of cajal are involved in neurotransmission in the gastrointestinal tract," Acta Histochemica et Cytochemica, vol. 39, no. 6, pp. 145-153, 2006.

[22] L. Johnston, C. Carson, A. D. Lyons, R. A. Davidson, and K. D. McCloskey, "Cholinergic-induced $\mathrm{Ca}^{2+}$ signaling in interstitial cells of Cajal from the guinea pig bladder," American Journal of Physiology-Renal Physiology, vol. 294, no. 3, pp. F645-F655, 2008.

[23] H.-N. Liu, S. Ohya, Y. Nishizawa et al., "Serotonin augments gut pacemaker activity via 5-HT3 receptors," PLoS One, vol. 6 , no. 9, 2011.

[24] J. N. Kim and B. J. Kim, "The mechanism of action of ghrelin and motilin in the pacemaker potentials of interstitial cells of cajal from the murine small intestine," Molecules and Cells, vol. 42, no. 6, pp. 470-479, 2019.

[25] J. Y. Jun, S. Choi, C. H. Yeum et al., "Noradrenaline inhibits pacemaker currents through stimulation of $\beta 1$-adrenoceptors in cultured interstitial cells of Cajal from murine small intestine," British Journal of Pharmacology, vol. 141, no. 4, pp. 670-677, 2004.

[26] S. Lee, H. Gim, J. H. Shim et al., "The traditional herbal medicine, Ge-Gen-Tang, inhibits pacemaker potentials by nitric oxide/cGMP dependent ATP-sensitive K+ channels in cultured interstitial cells of Cajal from mouse small intestine," Journal of Ethnopharmacology, vol. 170, pp. 201-209, 2015.
[27] J. J. Wu, T. P. Rothman, and M. D. Gershon, "Development of the interstitial cell of Cajal: origin, kit dependence and neuronal and nonneuronal sources of kit ligand," Journal of Neuroscience Research, vol. 59, no. 3, pp. 384-401, 2000.

[28] G. Radenkovic, V. Savic, D. Mitic, S Grahovac, M Bjelakovic, and M Krstic, "Development of c-kit immunopositive interstitial cells of Cajal in the human stomach," Journal of Cellular and Molecular Medicine, vol. 14, no. 5, pp. 1125-1134, 2010.

[29] K. M. Sanders, S. M. Ward, and S. D. Koh, "Interstitial cells: regulators of smooth muscle function," Physiological Reviews, vol. 94, no. 3, pp. 859-907, 2014.

[30] L.-M. Zhang, L.-J. Zeng, J. Deng et al., "Investigation of autophagy and differentiation of myenteric interstitial cells of Cajal in the pathogenesis of gastric motility disorders in rats with functional dyspepsia," Biotechnology and Applied Biochemistry, vol. 65, no. 4, pp. 533-539, 2018.

[31] X. Lin and J. Z. Chen, "Abnormal gastric slow waves in patients with functional dyspepsia assessed by multichannel electrogastrography," American Journal of Physiology-Gastrointestinal and Liver Physiology, vol. 280, no. 6, pp. G1370-G1375, 2001.

[32] S. Moraveji, M. Bashashati, S. Elhanafi et al., "Depleted interstitial cells of Cajal and fibrosis in the pylorus: novel features of gastroparesis," Neurogastroenterology \& Motility, vol. 28, no. 7, pp. 1048-1054, 2016.

[33] X.-J. Wang, J.-S. Guo, Y. Xu et al., "Effect of Shuwei decoction on rats with functional dyspepsia," Chinese Journal of Integrative Medicine, 2016.

[34] J. J. Rumessen, J.-M. Vanderwinden, and T. Horn, “Crohn's disease: ultrastructure of interstitial cells in colonic myenteric plexus," Cell and Tissue Research, vol. 344, no. 3, pp. 471-479, 2011.

[35] N. Bernardini, C. Segnani, C. Ippolito et al., "Immunohistochemical analysis of myenteric ganglia and interstitial cells of Cajal in ulcerative colitis," Journal of Cellular and Molecular Medicine, vol. 16, no. 2, pp. 318-327, 2012.

[36] A. E. Feldstein, S. M. Miller, M. El-Youssef et al., "Chronic intestinal pseudoobstruction associated with altered interstitial cells of Cajal networks," Journal of Pediatric Gastroenterology and Nutrition, vol. 36, no. 4, pp. 492-497, 2003.

[37] T. Suzuki, K.-J. Won, K. Horiguchi et al., "Muscularis inflammation and the loss of interstitial cells of Cajal in the endothelin ETB receptor null rat," American Journal of Physiology-Gastrointestinal and Liver Physiology, vol. 287, no. 3, pp. G638-G646, 2004.

[38] M. Kiyosue, M. Fujisawa, K. Kinoshita, M. Hori, and H. Ozaki, "Different susceptibilities of spontaneous rhythmicity and myogenic contractility to intestinal muscularis inflammation in the hapten-induced colitis," Neurogastroenterology and Motility, vol. 18, no. 11, pp. 1019-1030, 2006.

[39] S.-L. Fu, L.-N. Zhu, H.-H. Yin, Y. Jiang, and J.-C. Zhu, “Dacheng-qi decoction (大承气汤) combined with lactobacillus acidophilus improves gastrointestinal function of traumatic brain-injured model mice," Chinese Journal of Integrative Medicine, vol. 26, no. 10, pp. 762-768, 2020.

[40] Y.-C. Dai, Z.-P. Tang, Z.-N. Wang, Y.-L. Zhang, and X.-Y. He, "Influence of shenqing recipe on morphology and quantity of colonic interstitial cells of cajal in trinitrobenzene sulfonic acid induced rat colitis," Chinese Medical Sciences Journal, vol. 26, no. 1, pp. 43-48, 2011.

[41] J. H. Shim, S. J. Lee, H. Gim et al., "Regulation of the pacemaker activities in cultured interstitial cells of Cajal by 
Citrus unshiu peel extracts," Molecular Medicine Reports, vol. 14, no. 4, pp. 3908-3916, 2016.

[42] H. J. Kim, T. Han, Y. T. Kim, I. So, and B. J. Kim, "Magnolia officinalis bark extract induces depolarization of pacemaker potentials through M2 and M3 muscarinic receptors in cultured murine small intestine interstitial cells of cajal," Cellular Physiology and Biochemistry, vol. 43, no. 5, pp. 1790-1802, 2017.

[43] S.-K. Sung, S. J. Kim, T. S. Ahn et al., "Effects of Dangkwisoosan, a traditional herbal medicine for treating pain and blood stagnation, on the pacemaker activities of cultured interstitial cells of Cajal," Molecular Medicine Reports, vol. 12, no. 4, pp. 6370-6376, 2015.

[44] J. N. Kim, J. H. Nam, J. R. Lee, S. C. Kim, and B. J. Kim, “The traditional medicine banhasasim-tang depolarizes pacemaker potentials of cultured interstitial cells of cajal through M3 muscarinic and 5-HT3 receptors in murine small intestine," Digestion, vol. 101, no. 5, pp. 536-551, 2020.

[45] H. Kim, H. Kim, D. Yang, M. Jung, and B. Kim, "Depolarizing effects of daikenchuto on interstitial cells of cajal from mouse small intestine," Pharmacognosy Magazine, vol. 13, no. 49, pp. 141-147, 2017.

[46] H. J. Kim, G. S. Lee, H. Kim, and B. J. Kim, "Hwangryunhaedok-tang induces the depolarization of pacemaker potentials through 5-HT3and 5-HT4receptors in cultured murine small intestine interstitial cells of Cajal," World Journal of Gastroenterology, vol. 23, no. 29, pp. 5313-5323, 2017.

[47] B. J. Kim, H. Kim, G. S. Lee, I. So, and S. J. Kim, "Effects of San-Huang-Xie-Xin-tang, a traditional Chinese prescription for clearing away heat and toxin, on the pacemaker activities of interstitial cells of Cajal from the murine small intestine," Journal of Ethnopharmacology, vol. 155, no. 1, pp. 744-752, 2014.

[48] M. W. Hwang, H. J. Lee, H. J. Song, and B. J. Kim, "Involvement of MAPKs and PLC pathways in modulation of pacemaking activity by So-Cheong-Ryong-Tang in interstitial cells of cajal from murine small intestine," The Scientific World Journal, vol. 2013, Article ID 536350, 10 pages, 2013.

[49] J. N. Kim, J. H. Nam, J. R. Lee, S. C. Kim, Y. K. Kwon, and B. J. Kim, "Rikkunshito depolarizes pacemaker potentials of cultured interstitial cells of cajal through ghrelin receptors in murine small intestine," Digestion, vol. 101, no. 3, pp. 227-238, 2020.

[50] B. J. Kim, "Shengmaisan regulates pacemaker potentials in interstitial cells of cajal in mice," Journal of Pharmacopuncture, vol. 16, no. 4, pp. 36-42, 2013.

[51] J. N. Kim, H. J. Song, B. Lim, Y. K. Kwon, and B. J. Kim, "Modulation of pacemaker potentials by Pyungwi-san in interstitial cells of cajal from murine small intestine-pyungwi-san and interstitial cells of cajal," Journal of Pharmacopuncture, vol. 16, no. 1, pp. 43-49, 2013.

[52] M. Hwang, J. N. Kim, and B. J. Kim, "Hesperidin depolarizes the pacemaker potentials through 5-HT4 receptor in murine small intestinal interstitial cells of Cajal," Animal Cells and Systems, vol. 24, no. 2, pp. 84-90, 2020.

[53] Y.-J. Jeon, J.-S. Lee, Y.-R. Cho et al., "Banha-Sasim-Tang improves gastrointestinal function in loperamide-induced functional dyspepsia mouse model," Journal of Ethnopharmacology, vol. 238, Article ID 111834, 2019.

[54] N. Jiang, Y. Yu, F.-Q. Chen, L.-L. Yuan, and Q.-M. Wang, "Effect of banxia xiexin decoction on gastric antral interstitial cells of Cajal and stem cell factor in diabetic rats," Zhongguo Zhong Xi Yi Jie He Za Zhi, vol. 33, no. 12, pp. 1672-1676, 2013.
[55] Q. Zhao, F. Xing, Y. Tao et al., "Xiaozhang Tie improves intestinal motility in rats with cirrhotic ascites by regulating the stem cell factor/c-kit pathway in interstitial cells of cajal," Frontiers in Pharmacology, vol. 11, no. 1, 2020.

[56] S. Yan, Y.-Z. Yue, X.-P. Wang et al., "Aqueous extracts of Herba Cistanche promoted intestinal motility in loperamideinduced constipation rats by ameliorating the interstitial cells of cajal," Evidence-Based Complementary and Alternative Medicine, vol. 2017, Article ID 6236904, 13 pages, 2017.

[57] J. Yin, Y. Liang, D. Wang et al., "Naringenin induces laxative effects by upregulating the expression levels of c-Kit and SCF, as well as those of aquaporin 3 in mice with loperamideinduced constipation," International Journal of Molecular Medicine, vol. 41, no. 2, pp. 649-658, 2018.

[58] X. Zhou, H. Qian, D. Zhang, and L. Zeng, "Inhibition of autophagy of Cajal mesenchymal cells by gavage of tong bian decoction based on the rat model of chronic transit constipation," Saudi Journal of Biological Sciences, vol. 27, no. 2, pp. 623-628, 2020.

[59] S. Yan, Y.-Z. Yue, M.-M. Sun, B.-S. Wu, and X.-P. Wang, "Suppressive effect of Aurantii Fructus immaturus and atractylodis Macrocephalae rhizoma on glutamic acid-induced autophagy of interstitial cells of cajal," Journal of Integrative Medicine, vol. 18, no. 4, pp. 334-343, 2020.

[60] Y.-C. Dai, L. Zheng, Y.-L. Zhang et al., "Jianpi Qingchang decoction regulates intestinal motility of dextran sulfate sodium-induced colitis through reducing autophagy of interstitial cells of Cajal," World Journal of Gastroenterology, vol. 23, no. 26, pp. 4724-4734, 2017.

[61] R. Q. Tan, Z. Zhang, J. Ju, and J. H. Ling, "Effect of Chaihu Shugan powder-contained serum on glutamate-induced autophagy of interstitial cells of cajal in the rat gastric antrum," Evidence-based Complementary and Alternative Medicine, vol. 2019, Article ID 7318616, 7 pages, 2019.

[62] Y. H. Kwon, N. Kim, R. H. Nam et al., "Change in the interstitial cells of cajal and nNOS positive neuronal cells with aging in the stomach of F344 rats," PLoS One, vol. 12, no. 1, Article ID e0169113, 2017.

[63] N. Kaji, S. Nakayama, K. Horiguchi, S. Iino, H. Ozaki, and M. Hori, "Disruption of the pacemaker activity of interstitial cells of Cajal via nitric oxide contributes to postoperative ileus," Neurogastroenterology \& Motility, vol. 30, no. 8, Article ID e13334, 2018.

[64] N. Kaji, K. Horiguchi, S. Iino et al., "Nitric oxide-induced oxidative stress impairs pacemaker function of murine interstitial cells of Cajal during inflammation," Pharmacological Research, vol. 111, pp. 838-848, 2016.

[65] H. Salmhofer, W. Neuhuber, P. Ruth et al., "Pivotal role of the interstitial cells of Cajal in the nitric oxide signaling pathway of rat small intestine," Cell and Tissue Research, vol. 305, no. 3, pp. 331-340, 2001.

[66] P. Pacher, J. S. Beckman, and L. Liaudet, "Nitric oxide and peroxynitrite in health and disease," Physiological Reviews, vol. 87, no. 1, pp. 315-424, 2007.

[67] H. Chen, T. Ördög, J. Chen et al., "Differential gene expression in functional classes of interstitial cells of Cajal in murine small intestine," Physiological Genomics, vol. 31, no. 3, pp. 492-509, 2007.

[68] S. A. Means and L. K. Cheng, "Mitochondrial calcium handling within the interstitial cells of Cajal," American Journal of Physiology-Gastrointestinal and Liver Physiology, vol. 307, no. 1, pp. G107-G121, 2014.

[69] S. M. Ward, T. Ördög, S. D. Koh et al., "Pacemaking in interstitial cells of Cajal depends upon calcium handling by 
endoplasmic reticulum and mitochondria," The Journal of Physiology, vol. 525, no. 2, pp. 355-361, 2000.

[70] I. Khan, S. E. Samson, and A. K. Grover, "Antioxidant supplements and gastrointestinal diseases: a critical appraisal," Medical Principles and Practice, vol. 26, no. 3, pp. 201-217, 2017.

[71] S. Sen and R. Chakraborty, "Herbs, gastrointestinal protection, and oxidative stress," in Gastrointestinal Tissue, J. Gracia-Sancho and J. Salvadó, Eds., Academic Press, Cambridge, MA, USA, pp. 259-274, 2017.

[72] C. Lauridsen, "From oxidative stress to inflammation: redox balance and immune system," Poultry Science, vol. 98, no. 10, pp. 4240-4246, 2019.

[73] Q.-H. Jin, H.-X. Shen, H. Wang, Q.-Y. Shou, and Q. Liu, "Curcumin improves expression of SCF/c-kit through attenuating oxidative stress and NF- $\kappa \mathrm{B}$ activation in gastric tissues of diabetic gastroparesis rats," Diabetology \& Metabolic Syndrome, vol. 5, no. 1, p. 12, 2013.

[74] F. C. Vieira Frez, J. V. Martins Colombo Perles, D. Robert Linden et al., "Restoration of density of interstitial cells of Cajal in the jejunum of diabetic rats after quercetin supplementation," Revista Espanola de Enfermedades Digestivas, vol. 109, no. 3, pp. 190-195, 2017.

[75] T. Ordög, "Interstitial cells of Cajal in diabetic gastroenteropathy," Neurogastroenterology \& Motility, vol. 20, no. 1, pp. 8-18, 2008.

[76] B. J. Kim and B. Kuo, "Gastroparesis and functional dyspepsia: a blurring distinction of pathophysiology and treatment," Journal of Neurogastroenterology and Motility, vol. 25, no. 1, pp. 27-35, 2019.

[77] K. M. Sanders and S. M. Ward, "Interstitial cells of Cajal: a new perspective on smooth muscle function," The Journal of Physiology, vol. 576, no. 3, pp. 721-726, 2006.

[78] J. D. Huizinga, N. Zarate, and G. Farrugia, "Physiology, injury, and recovery of interstitial cells of cajal: basic and clinical science," Gastroenterology, vol. 137, no. 5, pp. 1548-1556, 2009.

[79] S.-J. Ko, S.-H. Cho, K.-J. Kim, J.-S. Kim, N.-Y. Ha, and J.-W. Park, "Herbal medicine Banha-Sasim-Tang for the treatment of functional dyspepsia protocol for a systematic review of randomized controlled trials," Medicine, vol. 98, no. 22, Article ID e15760, 2019.

[80] S. Mogami and T. Hattori, "Beneficial effects of rikkunshito, a Japanese kampo medicine, on gastrointestinal dysfunction and anorexia in combination with western drug: a systematic review," Evidence-Based Complementary and Alternative Medicine, vol. 2014, Article ID 519035, 7 pages, 2014.

[81] H. Miwa, M. Kusano, T. Arisawa et al., "Evidence-based clinical practice guidelines for functional dyspepsia," Journal of Gastroenterology, vol. 50, no. 2, pp. 125-139, 2015.

[82] J. W. Park, S. J. Ko, G. Han, I. Yeo, B. Ryu, and J. Kim, "The effects of banha-sasim-tang on dyspeptic symptoms and gastric motility in cases of functional dyspepsia: a randomized, double-blind, placebo-controlled, and two-center trial," Evidence-based Complementary and Alternative Medicine, vol. 2013, Article ID 265035, 10 pages, 2013.

[83] L. Zhao, S. Zhang, Z. Wang et al., "Efficacy of modified ban Xia Xie Xin decoction on functional dyspepsia of cold and heat in complexity syndrome: a randomized controlled trial," Evidence-based Complementary and Alternative Medicine, vol. 2013, Article ID 812143, 8 pages, 2013.

[84] Y. Gan, H. Liu, L. Yang, and K. Yang, "Effect of banxiaxiexin tang on treatment of functional dyspepsia: a meta-analysis of randomized controlled trials," Journal of Traditional Chinese Medicine, vol. 34, no. 2, pp. 140-144, 2014.

[85] K. Tominaga, Y. Sakata, H. Kusunoki et al., "Rikkunshito simultaneously improves dyspepsia correlated with anxiety in patients with functional dyspepsia: a randomized clinical trial (the DREAM study)," Neurogastroenterology \& Motility, vol. 30, no. 7, Article ID e13319, 2018.

[86] M. Arai, T. Matsumura, N. Tsuchiya et al., "Rikkunshito improves the symptoms in patients with functional dyspepsia, accompanied by an increase in the level of plasma ghrelin," Hepatogastroenterology, vol. 59, no. 113, pp. 62-66, 2012.

[87] S. J. Ko, J. Park, M. j. Kim, J. Kim, and J. W. Park, "Effects of the herbal medicine Rikkunshito, for functional dyspepsia: a systematic review and meta-analysis," Journal of Gastroenterology and Hepatology, vol. 36, no. 1, pp. 64-74, 2021.

[88] T. Matsumura, M. Arai, Y. Yonemitsu et al., "The traditional Japanese medicine Rikkunshito increases the plasma level of ghrelin in humans and mice," Journal of Gastroenterology, vol. 45, no. 3, pp. 300-307, 2010.

[89] H. Suzuki, J. Matsuzaki, Y. Fukushima et al., "Randomized clinical trial: Rikkunshito in the treatment of functional dyspepsia-a multicenter, double-blind, randomized, placebocontrolled study," Neurogastroenterology \& Motility, vol. 26, no. 7, pp. 950-961, 2014.

[90] M. W. Hwang, J. N. Kim, H. J. Song et al., "Effects of Lizhong Tang on cultured mouse small intestine interstitial cells of Cajal," World Journal of Gastroenterology, vol. 19, no. 14, pp. 2249-2255, 2013.

[91] H. Gim, J. H. Nam, S. Lee et al., "Quercetin inhibits pacemaker potentials via nitric oxide/cGMP-dependent activation and TRPM7/ANO1 channels in cultured interstitial cells of cajal from mouse small intestine," Cellular Physiology and Biochemistry, vol. 35, no. 6, pp. 2422-2436, 2015.

[92] H. Tian, D. Huang, T. Li et al., "The protective effects of total phenols in magnolia officinalix rehd. et wils on gastrointestinal tract dysmotility is mainly based on its influence on interstitial cells of cajal," International Journal of Clinical and Experimental Medicine, vol. 8, no. 11, pp. 20279-20286, 2015.

[93] Y. Zhang, H. Zhang, K. Zhang et al., "Co-hybridized composite nanovesicles for enhanced transdermal eugenol and cinnamaldehyde delivery and their potential efficacy in ulcerative colitis," Nanomedicine: Nanotechnology, Biology and Medicine, vol. 28, Article ID 102212, 2020.

[94] C. Peng, L. Wang, Y.-H. Wang, Y.-X. Li, and Y. Pan, "The toxicity of aconitine, emodin on ICC cell and the antagonist effect of the compatibility," European Journal of Drug Metabolism and Pharmacokinetics, vol. 34, no. 3-4, pp. 213-220, 2009.

[95] Y.-J. Xiong, D.-P. Chen, B.-C. Lv, F.-F. Liu, L. Wang, and Y. Lin, "Characteristics of nobiletin-induced effects on jejunal contractility,” Fitoterapia, vol. 94, pp. 1-9, 2014.

[96] Z. Yao, W. Namkung, E. A. Ko et al., "Fractionation of a herbal antidiarrheal medicine reveals eugenol as an inhibitor of $\mathrm{Ca}^{2+}$-activated $\mathrm{Cl}^{-}$channel TMEM16A," PLoS One, vol. 7, no. $5,2012$. 